\title{
Ethnicity, Obesity, and Type 2 Diabetes of Adults in Urban Populations of Central America
}

\author{
William G. Wuenstel ${ }^{1}$, James A. Johnson², James Humphries ${ }^{3}$, Cheryl Samuel ${ }^{4}$ \\ ${ }^{1}$ CMU Global Campus Instructor and Medical Social Scientist, USA \\ 2,3,4 Central Michigan University, Professor, Medical Social Scientist and Visiting Professor, St. Georges University, \\ Grenada, West Indies, USA
}

\begin{tabular}{l} 
Article Info \\
\hline Article history: \\
Received Dec 12, 2016 \\
Revised Feb 13, 2017 \\
Accepted Feb 25, 2017 \\
\hline
\end{tabular}

\section{Keyword:}

Type 2 Diabetes

Obesity

Ethnicity

Central America

\begin{abstract}
The purpose of this meta-analysis was to examine the impact of ethnicity and obesity as it relates to Type-2 Diabetes (T2D) in specific Central American countries. A meta-analysis was conducted to determine the association of ethnicity, obesity, and T2D. Four studies that qualified for inclusion were identified by searching MEDLINE and PubMed databases. The studies on the association of ethnicity and T2D had a combined population resulted in 265,858 study participants. Two studies on the association of obesity and T2D had 197,899 participants. An analysis of the data was conducted utilizing the relative risk ration, odds ratio, and forest plots. The comparison of the relative risk of T2D across ethnic categories by studies range for Blacks was 1.59 to 2.74, Asians was 1.43 to 2.08, and Hispanics .92 to 2.91. The ethnic difference in the prevalence of diabetes was almost two-fold higher in all ethnic groups than among the Caucasians with a significance level of $95 \%$. A comparison of relative risk of T2D across weight categories was significantly higher among those with a diagnosed of diabetes in all reported areas. The odds ratio was very close to the risk ratio in both ethnicity and obesity to the development of T2D.The meta-analysis findings documented that an association does exist between ethnicity and obesity to the development of type 2 diabetes.
\end{abstract}

Copyright $\odot 2017$ Institute of Advanced Engineering and Science. All rights reserved.

\section{Corresponding Author:}

William G. Wuenstel

Global Campus Instructor,

Central Michigan University,

1204 Health Professions Building, Mount Pleasant, Michigan, USA.

Email: wuens1wg@cmich.edu

\section{INTRODUCTION}

In a survey of the research, it was revealed that the number of people with diabetes mellitus (DM) [Type 1, Type 2 and Gestational] is growing. In 1990, DM was estimated to be the 29th leading cause of disease globally, rivaling respiratory infections and malignant neoplasm [1]. Twenty-three years later, it has become the fourth leading cause of death in most affluent countries, and there is significant evidence that it is epidemic levels in many economically emerging and newly industrialized nations [2]. Increases in population life expectancies and trends towards urbanization have contributed to the growing epidemic [3]. In addition, nutrition, obesity, and physical inactivity are recognized as contributing factors to diabetes. Current global projections report 382 million people are diagnosed with diabetes and a reported estimate of 1.5 million deaths in 2012 [4]. Experts also project the number of deaths to double between 2005 and 2030 [5]. The International Diabetes Federation (IDF), reported the global burden, explaining that the majority of people with diabetes are between the ages of 40 and 59, with $80 \%$ of them living in the low and middle-income countries [6]. 


\section{METHOD}

The primary objective of our study was to perform a meta-analysis of the literature addressing the association of ethnicity and obesity to T2D in a further effort to summarize the characteristics of the heterogeneous disorder [7]. A MEDLINE search of articles with a publication date from 1992 through 2014 containing the words "type 2 diabetes and ethnicity," and "type 2 diabetes and obesity," returned 16 articles for possible inclusion.

\subsection{Study selection}

Because meta-analysis focuses on aggregation and comparison of findings from different research studies, it will be necessary to sort studies that can be meaningfully compared. Therefore, the findings included in the meta-analysis will need to be conceptually comparable and include similar constructs and relationships. To be included, studies had to be prospective cohort studies and cross-sectional studies that included an individual measure of the variables being studied. For example, the primary studies included in this project had the following inclusion criteria:

- Participants 21 years of age or older

- Participants with diagnosed type 2 diabetes

- Studies on obesity would include an individual measure of recorded BMI

- Ethnicity studies would include an individual measure of recorded ethnicity

\subsection{Selection criteria and definitions}

The following contains the detailed conditions and definitions of criteria that were utilized to select the primary studies that were included in the meta-analysis. Eligible studies in the meta-analysis will require involving patients diagnosed with T2D defined as the following: fasting plasma glucose of $\geq 126 \mathrm{mg} / \mathrm{dl}$ or an oral glucose tolerance test of $\geq 200 \mathrm{mg} / \mathrm{dl}$ [8]. Eligible studies on obesity in the meta-analysis will require having the BMI variable with the indicator of overweight $/$ obesity $\geq 25 \mathrm{~kg} / \mathrm{m}^{2}$ [8].

A number of selected studies included two-variable relationships and their association with type 2 diabetes while other studies compared a single variable and its association with the development of type 2 diabetes. Both two-group and single-group comparisons will be included in some studies. Multi-group studies compare the dependent variable with an independent variable such as ethnicity. Single group studies only compared pre- and post- risk assessment of data from the same group of subjects.

\subsection{Study characteristics of ethnicity}

Two studies met all of the inclusion criteria for ethnicity. Table 1 illustrates the study characteristics and outcomes. $\mathrm{T}$ he combined population resulted in 265,858 study participants. Although there were multiple independent variables (ethnicity, age, obesity,) including anthropometric measurements (BMI) and constructs in the methodology, only one outcome measurement (type 2 Diabetes) was employed for each study. One study followed a prospective cohort design while the other utilized a cross-sectional analysis of a baseline questionnaire at cohort entry.

Both studies originated in the United States and were affiliated with or sponsored by academic medical centers. The age of the participants ranged from 45 to 78, and the studies ranged from 3 years to 20 years. Both studies included Caucasian, African American, Asian, and Hispanic subjects, while one study included Native Hawaiians. The variable of type 2 diabetes was diagnosed with either a Glucose Tolerance Test (GTT) or fasting plasma glucose level in the studies.

Table 1. Characteristics of Studies Comparing the Incidence of Type 2 Diabetes and Ethnicity

\begin{tabular}{|c|c|c|c|c|c|c|c|}
\hline Study & Number & Age & Intake Measures & $\begin{array}{c}\text { Independent } \\
\text { Variable }\end{array}$ & $\begin{array}{c}\text { Dependent } \\
\text { Variable }\end{array}$ & Results & $\begin{array}{c}\text { Follow-up } \\
\text { years }\end{array}$ \\
\hline
\end{tabular}




\subsection{Study characteristics of obesity}

Table 2 presents the descriptive data of two studies selected in the association of obesity and type 2 diabetes. The studies consisted of several constructs and research studies of cross-sectional data from a national study while the other utilized a random-digit telephone survey conducted by the Centers for Disease Control and Prevention (CDC). Multiple variables were measured including age, gender, race, education level, and smoking status, as well as obesity and diabetes. The studies included 197,899 participants, consisting of both genders. The age of the participants ranged from 18 to 63.9 with a study range of 7 to 20 years. BMI was the accepted methods of measuring obesity. Each study categorized the BMI into normal, overweight, and obesity classes. Nguyen's study used weigh classes starting with BMI of 30.0 to 34.9 as class $1,35.0$ to 39.9 as class 2, and $\geq 40.0$ as class 3, while Mokdad et al.'s (2003) study omitted obese class 1. Nguyen et al.'s [11] study was affiliated with academic health centers, and Mokdad et al.'s [12] study was affiliated with the CDC.

\subsection{Data extraction and analysis}

Abstracted data included the study's characteristics such as the study citation, number of participants, length of the study and follow-up, independent and dependent variables, definition of measures, and results. Tables identifying the study characteristics were developed for the following variables: ethnicity and obesity.

Data analysis was performed utilizing the MIX 2.0 Professional software for meta-analysis. The MIX program is a valid tool for performing meta-analysis and may be particular useful in education environments [13]. MIX is the only program with a peer-reviewed and published validation report, which is accessible as public information on its website [14].

Table 2. Characteristics of Included Studies in Obesity and Type 2 Diabetes

\begin{tabular}{|c|c|c|c|c|c|c|c|}
\hline Study & Number & Age & $\begin{array}{c}\text { Intake } \\
\text { Measures }\end{array}$ & $\begin{array}{l}\text { Independent } \\
\text { Variable }\end{array}$ & $\begin{array}{l}\text { Dependent } \\
\text { Variable }\end{array}$ & Results & $\begin{array}{c}\text { Follow-up } \\
\text { Years }\end{array}$ \\
\hline $\begin{array}{l}\text { Nguyen et al. (2011). } \\
\text { Relationship } \\
\text { between obesity and } \\
\text { diabetes in the U.S. } \\
\text { adult population [11] }\end{array}$ & 2,894 & $20-63$ & $\begin{array}{c}\text { Data from } \\
\text { the } \\
\text { NHANES }\end{array}$ & $\begin{array}{c}\text { Obesity as } \\
\text { measured by } \\
\text { BMI }\end{array}$ & Type 2 DM & $\begin{array}{l}\text { The prevalence of } \\
\text { diabetes increases } \\
\text { with increase of } \\
\text { obesity }\end{array}$ & 7 \\
\hline $\begin{array}{c}\text { Mokdad et al. } \\
\text { (2003). Prevalence } \\
\text { of obesity, diabetes, } \\
\text { and obesity- } \\
\text { Related health risk } \\
\text { factors, 2001. U.S. } \\
\text { [12] }\end{array}$ & 195,005 & $>18$ & $\begin{array}{l}\text { BRFSS } \\
\text { telephone } \\
\text { interview }\end{array}$ & $\begin{array}{c}\text { Obesity as } \\
\text { measured by } \\
\text { BMI }\end{array}$ & Type 2 DM & $\begin{array}{l}\text { Increase of obesity } \\
\text { and diabetes in } \\
\text { both genders, all } \\
\text { ages, all races, all } \\
\text { educational levels, } \\
\text { and all smoking } \\
\text { levels. }\end{array}$ & 10 \\
\hline
\end{tabular}

\section{RESULTS AND ANALYSIS}

An analysis of the relative risk ratio was conducted on the ethnicity studies, which included the PAHO survey data from Central America and two studies that originated in the U.S. Relative risk (RR) is frequently utilized as a bivariate measure of the strength of an association between a factor and the occurrence of an event [15]. For example, in this case, the risk of developing a disease, such as diabetes, due to specific ethnicity versus individuals from another ethnicity can be used to compare the risk of developing the disease. An RR of 1 means that there is no difference in risk between two ethnic groups in developing the disease. An RR of $<1$ means that the development of diabetes is less likely to occur. An RR of $>1$ means that the development of diabetes is more likely to occur.

Table 3 displays the comparative results from the meta-analysis on ethnicity. The relative risk ratio for the Asian group higher in the PAHO study, but the survey questionnaire in several countries (Costa Rica and El Salvador) did not include ethnicity, and Guatemala only reported Hispanic data. The overall prevalence of diabetes was similar in both genders for all countries. In the Shai study, Blacks had a higher prevalence rate, but they were also reported as having a higher BMI and as well as being more sedentary than other ethnic groups. The Hispanic ethnic group followed by the Black ethnic group was higher in the Maskarinec study, and the study reported a smaller response rate of questionnaires in both of those groups. The relative risk ratios with a significance level of $95 \%$ for all three studies range for Blacks was 1.59 to 2.74, Asians was 1.43 to 2.08, and Hispanics 92 to 2.91 . The ethnic difference in the prevalence of diabetes was almost two-fold higher in all ethnic groups than among the Caucasians. 
Table 3. Comparison of the Relative Risk of T2D Across Ethnic Categories by Study

\begin{tabular}{lccccc}
\hline Study & No. Participants & White & Black & Asian & Hispanic \\
\hline PAHO & 10,822 & 1 & 1.59 & 2.08 & 0.92 \\
& & & $(1.48-1.70)$ & $(1.85-2.31)$ & $(.64-0.92)$ \\
Shai & 78,419 & 1 & 2.18 & 1.43 & 1.76 \\
& & & $(1.82-2.61)$ & $(1.08-1.90)$ & $(1.32-2.34)$ \\
Maskarinec & 187,439 & 1 & 2.74 & 1.74 & 2.91 \\
& & & $(2.61-2.88)$ & $(1.66-1.82)$ & $(2.77-3.04$ \\
\hline
\end{tabular}

An analysis of the relative risk ratio was conducted on the studies involving obesity as illustrated in Table 4. They included the PAHO survey data from Central America and two studies that originated in the U.S. In the PAHO study, the data on the prevalence of overweight/obesity was significantly higher among those with diagnosed diabetes in all reported areas. The mean weight, height, and BMI were higher among men than women in all sites. In both U.S. studies, the prevalence of obesity had increased as well as the prevalence of U.S. adults that were obese and had diabetes. This is an increase in reporting from previous studies. The Nguyen [11] study reported a clear association between obesity and diabetes with an increase in BMI. The Mokdad et al. [12] study shows a continuing increase of obesity and diabetes in genders, transcending all ages, races, education levels, and smoking levels.

Table 4. Comparison of Relative Risk of T2D across Weight Categories

\begin{tabular}{lllll}
\hline Study & No. Participants & Normal & Overweight & Obese \\
\hline PAHO & 10,822 & 1.0 & $2.09(1.85-2.33)$ & $4.54(3.87-5.21)$ \\
Nguyen & 21,205 & 1.0 & $1.64(1.47-1.81)$ & $2.51(2.26-2.76)$ \\
Mokdad & 195,005 & 1.0 & $1.59(1.46-1.73)$ & $3.44(3.17-3.74)$ \\
\hline
\end{tabular}

Table 5. Meta-Analysis on the Ethnic Comparison With the Development of T2D

\begin{tabular}{lllllll}
\hline Ethnic Comparison & Estimates & Mean & Median & Min & Max & SD \\
\hline Black-white & or(log) & 0.75 & 0.779 & 0.463 & 1.01 & 0.273 \\
& or & 2.117 & 2.179 & 1.589 & 2.746 & 1.314 \\
Asian-white & or(log) & 0.548 & 0.554 & 0.358 & 0.732 & 0.187 \\
& or & 1.730 & 1.740 & 1.430 & 2.079 & 1.206 \\
Hispanic-white & or(log) & 0.517 & 0.565 & -0.083 & 1.068 & 0.577 \\
& or & 1.677 & 1.759 & 0.920 & 2.910 & 1.781 \\
\hline
\end{tabular}

Table 6. Meta-Analysis of Obesity with Odds Ratio

\begin{tabular}{ccccccc}
\hline Comparison & Estimates & Mean & Median & Min & Max & SD \\
\hline Overweight - & or(log) & 0.565 & 0.49 & 0.463 & 0.0737 & 0.150 \\
Normal & or & 1.756 & 1.64 & 1.59 & 2.09 & 1.162 \\
Obese - Normal & or(log) & 1.222 & 1.235 & 0.920 & 1.512 & 0.297 \\
& or & 3.397 & 3.440 & 2.51 & 4.54 & 1.345 \\
\hline
\end{tabular}

The odds ratio is another measure of effect size by describing the strength of the association between two binary data values [15]. It is the ration of the odds of an event occurring in one group to the odds of it occurring in another group [15]. An odds ratio greater than 1 would indicate that the condition or event is more likely to occur in the first group [15]. In addition, an odd ratio less than 1 would indicate that the condition or event is less likely to occur in the first group [15]. Table 5 illustrates that Blacks, Asian, and Hispanic had a greater than 1 odd ratio for the development of T2D as opposed to the white ethnic group.

Table 6 presents the data on the odds ratio for obesity. Overweight population has a 1.756 chances in developing T2D than normal weight individuals. The obese group has greater than three times the risk of developing T2D as compared to normal weight individuals.

\subsection{Study limitations}

Meta-analysis has a number of advantages as a research method for its analytical tool as well as for summarizing research findings of other studies; however, it is not without limitations. Our study has several limitations and the results must be interpreted with caution. For example, possible limitations of this study on the association of ethnicity and obesity to the development of T2D include: (1) not all relevant studies may be located and included; (2) the inclusion of lower quality studies may dilute the strength of higher quality studies; (3) availability of data; (4) and the distortion in the results of the included studies from the use of various measurements. 
The third limitation had the greatest impact on the study due to the limited available data from Central America. Belize was the only country where the PAHO survey was conducted throughout the entire country and involved all ethnicities. The other Central American countries surveyed in specific urban areas. The last limitation involves the results of the numerous studies utilized in the meta-analysis. The attempt was made to find studies that compared "apples to apples" rather than "apples to oranges"-for example, studies that measured body mass index (BMI) rather than waist circumference (WC) or waist to hip ratio (WHR). However, most studies included multiple methods of obesity measurement. The studies on ethnicity contained measurements on various races and sub-groups such as Mestizo and Garifuna.

\subsection{Discussion}

This study provides additional insight into the relationship between ethnicity, obesity, and the development of T2D. The strength of the study includes the large sample size, long follow-up period, and extensive review of the literature. Number of participants for ethnicity was 276,680 and for obesity was 208,721 , and the follow-up period ranged from 3 years to 20 . The meta-analysis findings documented that an association does exist between ethnicity and obesity to the development of T2D. Studies from PAHO and several American studies have suggested that Blacks, Asians, and Hispanics have a greater risk of developing T2D in comparison to Caucasians. There was also a strong association of overweight and obesity to the development of T2D.

Studies from other parts of the world have shown an association of ethnicity, obesity to the development of T2D. China reported the number of people with diabetes (20-79 years) at 109.6 million as of 2015 and India reportedits total number as 69.2 million for the same period [6]. Both countries contribute this increase to the nutrition transition where traditional diets are being replaced with dietary intakes consisting of sugar, hydrogenate and saturated fats, and high-fat dairy products; all have been implicated in an increase of obesity and T2D [16]. In other Asian populations, the prevalence of T2D has greatly increase as well. For example, Indonesia is reporting 10 millions and Bangladesh has reported 7.1 million people with T2D [6]. Because of the these staggering numbers, Chan et al believe that greater than $60 \%$ of population of people with T2D will come from Asia [16]. Once considered a disease of the West where older populations are mostly affected, the burden of T2D in Asian countries will be disproportionately higher in the young to middle-age adults [17]. Similarly, other Asian countries are seeing an increase in overweight and obesity which is cause by the increase in socioeconomic development, nutrition transition, and the lack of physical activity, which promotes overnutrition and sedentary lifestyles [18]. The main cause of this influx is due to the younger Asian adults developing the metabolically obese phenotype which occurs when the adult has normal body weight with increased abdominal adiposity commonly seen in the Asian population [19]. This increases the risk of developing T2D diabetes at a lower BMI and age for Asians than for the Europeans [20]. These lifestyle changes, as we have seen in other countries, may contribute to the increasing number of diabetes especially in the young age group resulting in an increasing global epidemic that will not only cause devastating personal suffering but also an economic burden for every country around the world.

\section{CONCLUSION}

An important implication from this study is that T2D is largely preventable. Individuals cannot change their genes or ethnicity, but they can improve their food selections and increase their physical activity, as well as regulary monitoring their blood sugar. However, changes in behaviors will require a different model of care that places emphasis on primary health care and prevention as well as an increase in active surveillance. This study augments others that have been conducted by the Pan American Health Organization (PAHO) and researchers. In fact, this study states the critical nature of diabetes in the regions. All 20 countries in this region have similar age-distribution profiles with anexpected increased in the 50 years and older section. As urbanization continues and the populations grow older, diabetes will become an evergreater public health priority throughout the region.

While it has long been a desire of medical social science research to inform practice and policy, it does not always happen. However, this meta-analysis study does have the potential to do just that. It provides critical insights into a rapidly growing problem that has the potential to have a detrimental impact on the health and social well-being of an entire region.

\section{REFERENCES}

[1] S. Wild, et al., "Global burden of diabetes mellitus in the year 2000," 2000. [cited 2015 May 10]. Available from: www.who.int/entity/healthinfo/statistics/bod_diabetes.pdf 
[2] International Diabetes Federation, "Diabetes fact sheet," 2014. [cited 2015 May 10]. Available from: https://www.idf.org/webdata/docs/background_info_AFR.pdf

[3] S. Wild, et al., "Global prevalence of diabetes: Estimates for the year 2000 and projections for 2030," Diabetes Care, vol/issue: 27(5), pp.1047-1053, 2004.

[4] World Health Organization, "Diabetes Fact Sheet N312," 2016. [cited 2015 May 10]. Available from: http://www.who.int/mediacentre/factsheets/fs312/en/

[5] H. King, et al., "Global burden of diabetes, 1995-2025: Prevalence, numerical estimates, and projections," Diabetes Care, vol/issue: 21(9), pp. 1414-1431, 1998.

[6] International Diabetes Federation, "The diabetes atlas seventh edition," 2015. [cited 2015 May 10]. Available from: http://www.idf.org/sites/default/files/EN_6E_Atlas_Full_0.pdf

[7] Pan American Health Organization, "Regional Health Observatory leading causes of death," 2006. [cited 2015 May 11]. Available from: http://www.paho.org/hq/index.php?option=com_content\&view=article\&id=3501\&Itemid=2391

[8] Pan American Health Organization, "CAMDI Survey of diabetes, hypertension and chronic disease risk factors: Belize, San Jose, San Salvador, Guatemala City, Managua, and Tegucigalpa, 2012,” 2012.

[9] G. Maskarinec, et al., "Diabetes prevalence and body mass index differ by ethnicity: The Multiethnic Cohort," Ethnicity \& Disease, vol. 19, pp. 49-55, 2009.

[10] I. Shai, et al., "Ethnicity, obesity, and risk of type 2 diabetes in women," Diabetes Care, vol/issue: 29(7), pp.1585$1590,2006$.

[11] N. T. Nguyen, et al., "Relationship between obesity and diabetes in a US adult population: Findings from the National Health and Nutrition Examination Survey, 1999-2006," Obesity Surgery, vol. 21, pp. 351-355, 2011.

[12] A. H. Mokdad, et al., "Prevalence of obesity, diabetes and obesity-related health risk factors, 2001," JAMA, vol/issue: 289(1), 2003.

[13] L. Bax, et al., "Development and validation of MIX: comprehensive free software for meta-analysis of causal research data," BMC Medical Research Methodology, vol/issue: 6(50), pp. 1-11, 2006.

[14] L. Bax, et al., "A systematic comparison of software dedicated to meta-analysis of causal studies," BMC Medical Research Methodology, vol/issue: 7(40), pp. 1-9, 2007.

[15] L. Shi, "Health services research methods (2nd Edition)," Clifton Park, NY, Thomson Delm, 2008.

[16] A. Ramachandran, A. "Epidemiology of diabetes in India-Three decades of research," J Assoc Physicians India, vol. 53, pp. 34-38, 2005.

[17] J. Chan, et al., "Diabetes in Asia: Epidemiology, Risk Factors, and Pathophysiology," JAMA, vol/issue: 301(20), pp. 2129-2139, 2009.

[18] Misra A. and L. Khurana, "Obesity and the metabolic syndrome in developing countries," JClinical Endocrinology Metab, vol/issue: 93(11), pp. S9-S30.

[19] K. Yoon, et al., "Epidemic obesity and type 2 diabetes in Asia," Lancet, vol/issue: 368(9548), pp. 1681-1688, 2006.

[20] R. Huxley, et al., "Obesity in Asia Collaboration. Ethnic comparisons of the cross-sectional relationships between measures of body size with diabetes and hypertension," Obesity Review, vol/issue: 9(1), pp. 53-61, 2008. 